\title{
L'EDITIO PRINCEPS DES VARIAE DE CASSIODORE PAR MARIANGELUS ACCURSIUS ET LE MANUSCRIT 211 DE VALENCE (ESPAGNE)
}

\author{
Valérie Fauvinet-Ranson
}

\section{Le manuscrit de Valence et l'editio princeps des Variae}

En 1533 parut la première édition imprimée complète des Variae, à Augsbourg, sous le titre Magni Aurelii Cassiodori variarum libri XII, item de anima liber unus, recens inventi et in lucem dati a Mariangelo Accursio. La même année parut la première édition complète d'Ammien Marcellin: toutes deux étaient l'œuvre d'Accursius, Mariangelo Accursio, né à L'Aquila en 1489, précepteur de deux jeunes princes, les frères Johann Albrecht et Gumpert, marquis de Brandebourg, de la famille des Hohenzollern, venus parfaire leur éducation à Rome dans les années 1520. Avant cette édition des Variae, seuls étaient parus auparavant, en 1529, des extraits d'un choix de textes, publiés par Jean Cochlaeus $\left({ }^{1}\right)$ : ce dernier explique dans sa préface qu'il avait, au prix d'un travail laborieux, réuni les différents livres en un seul volume et copié le texte en comparant plusieurs manuscrits, puis laissé le tout à Rome, afin de pouvoir confronter son travail avec un manuscrit entier, objet rare, conservé in Vaticani secretiori bibliotheca, mais que le fruit de ce travail disparut durant le sac de la ville de 1527, ne lui laissant que le bref compendium qu'il publia.

L'editio princeps est donc celle d'Accursius et elle est importante, puisque c'est dans son texte qu'on a longtemps lu les Variae. Or Mommsen y a constaté l'existence de nombreuses leçons inconnues par ailleurs et qu'il n'a pu retrouver dans aucun des nombreux manuscrits qu'il a vus ou fait voir par d'autres. Aussi a-t-il mentionné ces leçons dans son apparatcritique sous la lettre A, comme Accursius $\left({ }^{2}\right)$. Accursius explique qu'il a trouvé le texte des Variae en Espagne, lors de son voyage à la suite de la cour de Charles Quint, toujours attaché aux jeunes princes allemands: «J'ai trouvé, tandis que je parcourais l'Espagne dans

(1) Sur ce volume, cf. Th. Mommsen, Cassiodori Senatoris Variae, Berlin 1894 (Monumenta Germaniae Historica, Auctores Antiquissimi, XII), p. Lxxxıv et cx. L'édition princeps d'Accursius peut ètre consultée sur le site de Google livres (books.google.fr) où le volume de l'Université Complutense de Madrid a été reproduit en mai 2009: l'adresse étant très longue et complexe, le plus simple est de taper "Magni Aurelii Cassiodori Variarum»sur Google et de parcourir les premières pages de résultats (démarche valable le 18/01/2011).

(2) $A^{r}$ représente un manuscrit très proche, Wroclaw, Rehdiger $62\left(=\mathrm{S}\right.$. I. $4.13, \mathrm{n}^{\circ} 51 \mathrm{de}$ Mommsen, MGH, op. cit.), écrit en 1358 à Avignon par un Padouan; quand il diverge de l'édition d'Accursius, celle-ci est alors nommée $A^{e}$. La lettre $A$ sans exposant désigne l'accord du manuscrit de Wroclaw et de l'édition d'Accursius, dans l'apparat de Mommsen. 
la suite de César, les livres des Variae» $\left({ }^{3}\right)$. Or, Mommsen n’a vu aucun des manuscrits qui sont conservés en Espagne, bien qu'il connaisse l'existence de la majorité d'entre eux $\left({ }^{4}\right)$. L'un, conservé depuis le XIX siècle à la bibliothèque universitaire de Valence (211), pourrait être celui d'Accursius (il sera désigné par la lettre $\beta$ dans la suite de cet article). Il se trouve en effet que les points communs entre ce manuscrit et le texte d'Accursius sont très nombreux et frappants. En voici une liste pour la préface et le livre I : elle serait beaucoup plus longue si je n'en avais exclu toutes les leçons communes avec certains des quelques vingt manuscrits que j'ai collationnés jusqu'à présent pour le livre $\left.\mathrm{I}^{5}{ }^{5}\right)$. On trouvera les sondages effectués dans les autres livres en annexe.

\begin{tabular}{|l|l|l|}
\hline Variae & Accursius et $\beta\left(^{6}\right)$ & Autres manuscrits vus $\left(^{7}\right)$ \\
\hline Préface, 9 & quaesumus quasi $\left(^{8}\right)$ & quaesumus \\
\hline $\mathrm{I}, 1,6$ & suspendatis a nobis $+W$ & suspendatis (ou suspendatis amplius) \\
\hline $\mathrm{I}, 2,3$ & sauciatis uix $+W$ & sauciatis (ou sauci(at)is bis) \\
\hline $\mathrm{I}, 2,4$ & albentes comas $+W$ & albentis comas \\
\hline $\mathrm{I}, 2,7$ & nam cum fame $+W$ & cum fame (ou ut fame) \\
\hline $\mathrm{I}, 2,7$ & ut est mos hominibus $+W$ & ut est hominibus (ou ut est moris/mox hominibus) \\
\hline $\mathrm{I}, 3,3$ & tui laboris & laboris tui \\
\hline $\mathrm{I}, 3,4$ & militauerunt & militauere \\
\hline $\mathrm{I}, 3,4$ & ubi solita $+W$ & ut solita \\
\hline $\mathrm{I}, 4,3$ & ad fastigium praeconii & ad praeconii fastigium \\
\hline $\mathrm{I}, 4,4$ & creuit tantum $+W$ & creuit \\
\hline
\end{tabular}

(3) Début de la Préface de l'édition: Inueni, dum in Comitatu Caesaris Hispaniam peragro, Variarum libros.

(4) Escorial, BRMSL, D. III. 21; Q. III. 18; A. II. 16. Il mentionne également un Hispanus Valentianus n. 71, qui correspond, par la description du contenu, au manuscrit 211 de la bibliothèque universitaire. Il ne connaissait ni l'existence d'un autre manuscrit de Valence, qui se trouve dans les archives de la cathédrale, 252 (183), un gros volume qui rassemble en fait deux manuscrits sur papier, ni celle du manuscrit de l'Escurial F. III. 18, que j'appellerai $\delta$. Je suis très reconnaissante à la bibliothèque universitaire de Valence de m'avoir procuré avec diligence un microfilm du manuscrit 211, ainsi que des photos et de m'avoir réservé sur place le meilleur accueil.

(5) Pour préparer l'édition de ce livre dans la Collection des Universités de France.

(6) Et parfois $\delta$ (cf. note 4), qui a été partiellement copié sur $\beta$ (cf. infra, «la postérité du manuscrit»). La mention $+W$ signale l'accord avec le manuscrit de Wroclaw cité en note 2, dont Mommsen a relevé les points communs avec $\beta$.

(7) Ce relevé repose sur les manuscrits utilisés par Mommsen dans son apparat (j'ai repris les lettres qu'il leur a données pour noms dans cette colonne et dans la suite de l'article); ils ont été revus et j'en ai collationné sept autres antérieurs à $\beta$ : si l'on reprend la numérotation de Mommsen, il s'agit des numéros 6 (Paris, BN, Lat. 2923); 12 (Cambridge, Trinity College Lib., 1341); 14 (Firenze, BML, Strozzi 023); 20 (Londres, British Lib., Royal 10. B. IV); 28 (Paris, BN, Lat. 2170); 32 (Paris, BN, Lat. 10609); 39-40 (Rouen, Bibl. mun., 490 (A. 360)); 104, (Vaticano, BAV, Chig. A. VII. 217). J'en ai vu d'autres qui sont plus tardifs que $\beta$ dont je parlerai plus loin.

(8) D'après les loci selecti de Mommsen, le manuscrit de Vienne, Oest. Nat., 924 comporte la même leçon. Il est notable que l'ordre des livres de ce manuscrit est semblable à celui de $\beta$, de mème que l'incipit. Cf. infra, note 32 pour la datation. 


\begin{tabular}{|c|c|c|}
\hline $\mathrm{I}, 4,5$ & uixit enim & uixit \\
\hline $\mathrm{I}, 4,7$ & sibi de se fecit $+W$ & sibi fecit \\
\hline $\mathrm{I}, 4,11$ & potentem armorum & armorum potentem \\
\hline $\mathrm{I}, 4,14$ & siciliam bruciosque & bruttios (ou autres orthographes) siciliamque \\
\hline $\mathrm{I}, 4,17$ & accipit & accepit \\
\hline $\mathrm{I}, 5,2$ & comitis ann (a)e iudicio & et in comitis ann(a)e iudicio \\
\hline $\mathrm{I}, 7,2$ & quae parentibus $+W$ & quod parentibus \\
\hline I, 8,2 & quia & et quia \\
\hline I, 8,2 & facias $+W$ & faciat \\
\hline I, 8,3 & uenire deproperet $+W$ & deproperet uenire \\
\hline $\mathrm{I}, 9,3$ & et equitatem & equitatem ou et probitatem \\
\hline $\mathrm{I}, 10,5$ & docta antiquitas & antiquitas docta \\
\hline $\mathrm{I}, 10,6$ & tanta & talia \\
\hline $\mathrm{I}, 10,7$ & et aliquid inde $+W$ & et aliquid \\
\hline $\mathrm{I}, 10,7$ & omnino & omnimodo \\
\hline $\mathrm{I}, 13,1$ & in uestrum coetum & in coetum uestrum \\
\hline I, 13,3 & sonantium & insonantium \\
\hline $\mathrm{I}, 13,3$ & multis & mutis \\
\hline $\mathrm{I}, 15,1$ & ut te sequentibus & ut sequentibus \\
\hline I, 15,2 & angeli & agne(l)li \\
\hline I, 16,2 & desideria & desidiam \\
\hline $\mathrm{I}, 16,2$ & $\begin{array}{l}\text { releuandam potius aestimemus } \\
\text { fortunam }\end{array}$ & $\begin{array}{l}\text { releuandam aestimemus esse fortunam ou rel. esse } \\
\text { aest. fort. }\end{array}$ \\
\hline I, 17,4 & prouidentiam habuisse & habuisse prouidentiam/prudentiam \\
\hline $\mathrm{I}, 18,2$ & $\begin{array}{l}\text { ingressus aduersus quam } \\
\text { praescriptio }\end{array}$ & ingressus quoniam praescriptio \\
\hline $\mathrm{I}, 19,1$ & debita nobis & nobis debita \\
\hline I, 19,2 & uobis praesenti $+W$ & praesenti uobis \\
\hline $\mathrm{I}, 19,2$ & quae debuit $+W$ & quod debuit \\
\hline I, 20,2 & insinuatione $+W$ & insinuata petitione \\
\hline $\mathrm{I}, 20,3$ & eorum utilitate & illorum utilitate \\
\hline I, 20,4 & theodero $\beta$ theodoro Acc & thorodon (et variantes) \\
\hline $\mathrm{I}, 21,2$ & Labor & si labor \\
\hline $\mathrm{I}, 21,3$ & $\begin{array}{l}\text { ibi se norunt refugere ubi lon- } \\
\text { gissima }\end{array}$ & ubi se norunt refugere longissima \\
\hline $\mathrm{I}, 22,2$ & etiam ipsa & ipsa etiam \\
\hline I, 22,3 & inquiramus $+W$ & inquirimus \\
\hline I, 22,4 & causa mala & mala causa \\
\hline $\mathrm{I}, 23,2$ & in eius quacumque parte $+W$ & in eius quoque parte \\
\hline I, 25,1 & non minor $+W$ & maior \\
\hline
\end{tabular}




\begin{tabular}{|c|c|c|}
\hline $\mathrm{I}, 25,1$ & custoditis autem $+W$ & custoditis \\
\hline I, 25,2 & Sicini $+W$ & Licini (ou Lucini ou Lucrini) \\
\hline I, 25,3 & formatas & tornatas (ou reuocatas) \\
\hline I, 25,3 & suas laudes & laudes suas \\
\hline I, 26,2 & uersilli(a)e & $\begin{array}{l}\text { unscil(a)e ou unscille ou unscilie ou uncile ou } \\
\text { uerscilae ou uersilie ou uerscilie ou uersile (et au- } \\
\text { tres variantes) }\end{array}$ \\
\hline I, 26,2 & cassiodori patricii & patricii cassiodori \\
\hline I, 27,2 & comitatum uenire & uenire comitatum \\
\hline $\mathrm{I}, 28,2$ & ciuitatis suae utilitati & utilitati suae ciuitatis \\
\hline $\mathrm{I}, 30,2$ & fide mala & mala fide \\
\hline $\mathrm{I}, 30,4$ & asserere & exerere (ou exercere) \\
\hline I, 30,5 & illi est prius inermem & est prius illi inermem \\
\hline I, 30,5 & fieri in ciuibus & in ciuibus fieri \\
\hline $\mathrm{I}, 32,4$ & fauorem migrauerit & migrauerit fauorem \\
\hline $\mathrm{I}, 33,1$ & patricios nostros $+W$ & patricios uiros \\
\hline $\mathrm{I}, 33,1$ & colligerent & eligerent \\
\hline I, 33,2 & decreuimus & decernimus \\
\hline I, 35,4 & torpedinis tactu & tactu torpedinis \\
\hline $\mathrm{I}, 37,5$ & angelo $+W$ & agnello \\
\hline $\mathrm{I}, 38,1$ & uidetur utile & utile uidetur \\
\hline $\mathrm{I}, 38,1$ & hilarii Acc hylarii $\beta$ & uuiliarit / uuiliarum (et variantes) \\
\hline I, 38,2 & potest captio & captio potest (potest cautio $\mathrm{K}$ ) \\
\hline $\begin{array}{l}\text { I, } 39,2 \text { in- } \\
\text { terpolation } \\
\text { d'un passage } \\
\text { de IV, } 6,3, \\
\text { comme dans } \\
K \text { et } F^{2}\end{array}$ & $\begin{array}{l}\text { impositum putet } \\
\text { facunda }\end{array}$ & $\begin{array}{l}\text { putet impositum }\left(K F^{2}\right) \\
\text { fecunda }\left(K F^{2}\right)\end{array}$ \\
\hline I, 39,2 & forsitan & forte \\
\hline I, 40 & non debet per moram & per moram non debet \\
\hline I, 40 & cum ea tempus $+W$ & cum tempus \\
\hline I, 40 & necessaria fuerit & fuerit necessaria \\
\hline I, 41 & uolumus numero & numero uolumus \\
\hline I, 41 & haec enim $+W$ & hoc enim (ou hic est $E$ ) \\
\hline I, 42,1 & initium & in tutum (ou intuitus $K$ ) \\
\hline I, 42,1 & omnis reponit $+W$ & reponit (ou deponit $P /$ deponerit $D$ ) omnis \\
\hline $\mathrm{I}, 43,1$ & gaudium $+W$ & genium (ou geminum $K$ ) \\
\hline I, 43,1 & quod Acc quo $\beta^{a}$ & quicquid \\
\hline I, 44, 1 & haberent innoxii & haberent et innoxii \\
\hline I, 45,1 & oblectamina Acc oblectamenia $\beta$ & oblectamenta \\
\hline
\end{tabular}




\begin{tabular}{|l|l|l|}
\hline $\mathrm{I}, 45,2$ & nobis est $+W$ & nobis \\
\hline $\mathrm{I}, 45,5$ & monstrare & miracula monstrare \\
\hline $\mathrm{I}, 45,5$ & iniuncta $+W$ & minuta (ou musica) \\
\hline $\mathrm{I}, 45,6$ & propriam uocem & uocem propriam \\
\hline $\mathrm{I}, 45,7$ & enim fecit & fecit \\
\hline $\mathrm{I}, 45,10$ & hoc... hoc... hoc & haec... haec... haec \\
\hline $\mathrm{I}, 45,11$ & comperimus & cognouimus \\
\hline
\end{tabular}

\section{Histoire du manuscrit de Valence}

Avant de commenter ces points communs, il convient de vérifier si Accursius a pu avoir accès à ce manuscrit. On sait que son séjour en Espagne fut long et qu'il dura de 1525 à 1529. $\beta$ se trouvait-il déjà en Espagne à cette date-là ? Il semble que oui : on le sait grâce à l'inventaire qui a été rédigé, en 1527 à Ferrare, des livres hérités de son père par Ferdinand d'Aragon, duc de Calabre: la plus grande partie de la bibliothèque des rois de Naples aragonais lui avait échappé et était arrivée en France, soit dans le butin de Charles VIII («unze cent quarante livres de toutes sortes apportés de Napples» $\left({ }^{9}\right)$ ), soit à la suite de ventes effectuées en faveur du cardinal d'Amboise $\left({ }^{10}\right)$ et de Louis XII $\left({ }^{11}\right)$ par la reine Isabelle, la mère de Ferdinand, ruinée à la mort de son mari, l'ex-roi de Naples Frédéric, en 1504. Toutefois, une partie de la bibliothèque resta en la possession d'Isabelle, réfugiée à Ferrare. Son fils, nommé vice-roi de Valence en 1524, décida de faire venir ces livres dans cette ville et en fit dresser l'inventaire, qui a été retrouvé et acquis en 1983 par la bibliothèque universitaire de Valence $\left({ }^{12}\right)$. Sous le numéro 89 y figure un volume de Cassiodore:

«Cassiodoro de le degnità de la ecclesia romana, et con uno trattato de anima, de le dignità consulari de Roma. De volume de foglio comune, scripto de littera formata bastarda; miniato con un friso. Comenza de littere rosse Magni Aurelii Cassiodoris (sic) senatoris, viri clarissimi et illustris questoris palacio officiorum, et in fine de la tavola oratio quam fecit. Rubrica CLXXXIII. Coperto de coiro verde, con chiudende de rame. Signato Cassiodoro 3; notato alo imballaturo a ff. 232, partita $2^{\mathrm{a}} . »\left({ }^{13}\right)$

S'agit-il bien de notre manuscrit ? On pourrait en douter: les morceaux de l'ancienne reliure qui sont intégrés dans une couverture plus récente sur laquelle on a gardé d'anciens

(9) «Declaration des livres en latin et en français, italien, grec et esbrieu appartenant a la Royne Duchesse <Anne de Bretagne>» du 7 septembre 1498 (T. De Marinis, La Biblioteca Napoletana dei re d'Aragona, Milan, 1947-1952, I, p. 200, n. 8), et P. Cherchi et T. De Robertis, «Un inventario della Biblioteca Aragonese», Italia medioevale e umanistica, XXXIII, 1990, pp. 109-347, p. 109.

(10) Entre 1502 et 1504, T. De Marinis, La Biblioteca (supra n. 9), I, p. 201, n. 15, l. 4, et P. Cherchi et T. De Robertis, "Un inventario» (supra n. 9), p. 109.

(11) T. De Marinis, La Biblioteca (supra n. 9), I, p. 195 et n. 11, et P. Cherchi et T. De RoBERTis, "Un inventario» (supra n. 9) p. 109.

(12) P. Cherchi et T. De Robertis, "Un inventario» (supra n. 9), p. 111. Manuscrit 947 de la bibliothèque universitaire (f. 62r-135r).

(13) P. Cherchi et T. De Robertis, «Un inventario» (supra n. 9), p. 176. La cote actuelle de la bibliothèque universitaire indiquée par eux semble erronée: c'est 211, et non 221. Celle qu'ils 
fermoirs de cuivre sont de cuir brun et non vert; mais il a pu perdre sa couleur et le décor de cette ancienne couverture, de style mudejar, ressemble de façon frappante à celui des reliures de la bibliothèque aragonaise de Naples $\left({ }^{14}\right)$.

Quant à l'incipit de notre manuscrit, il est identique en son début avec celui qu'a relevé l'inventaire, mais il en diffère par les derniers mots (voir pl. 40):

«magni aurelii cassiodori senatoris viri clarissimi et illustris quaestoris palatii consulis ordinarii prepositi officiorum» (et non «palacio officiorum»).

Toutefois, ces deux incipit ont en commun le mot quaestoris au lieu de ex quaestoris que l'on trouve dans la plupart des manuscrits des Variae. On peut donc supposer que c'est le même incipit, qui aurait été simplifié sur la fin par le rédacteur de l'inventaire. La ressemblance des deux explicit est encore plus frappante: dans le manuscrit $\beta$, au folio 184, on lit effectivement oratio quam facit rubrica CLXXIII : les deux petites différences par rapport à la citation du catalogue (fecit et CLXXXIII) peuvent être des erreurs de copie. Plusieurs éléments concordent donc et permettent de supposer que $\beta$ est le manuscrit qui figure dans l'inventaire de 1526 et cette identité est admise sans discussion par ceux qui se sont intéressés à la bibliothèque des rois d'Aragon $\left({ }^{15}\right)$.

L'examen du décor de la première page confirme le passage de $\beta$ par la cour de Naples, bien qu'il ne figure pas d'armes dans l'écu soutenu par deux putti qui se trouve en bas. Aucun catalogue, à ma connaissance, ne mentionne le fait que ce décor en remplace un autre à filigranes et antennes qui a été gratté, mais dont il subsiste des arabesques et des œufs de grenouille le long de la majuscule ornée, ce qui apparaît nettement quand on examine le manuscrit lui-mème ou une bonne photo (voir pl. 40). C'est ce type de décor qui subsiste partout ailleurs (voir pl. 41-42): seule la première page a été re-décorée, au moment de l'entrée du manuscrit dans la bibliothèque royale de Naples, peut-on supposer, sur le modèle de la plupart des autres manuscrits, mais de façon moins somptueuse $\left({ }^{16}\right)$.

D'où provenait ce manuscrit? Il pourrait avoir été écrit au XIV siècle, en Italie centrale ou à Bologne $\left({ }^{17}\right)$. On ne sait pas quand il est entré dans la bibliothèque aragonaise. Il ne figure en effet pas dans l'inventaire de la bibliothèque de Ferdinand I $^{\text {er }}$ d'Aragon, rédigé en

indiquent pour le catalogue de Marcelino Gutiérrez del Caño, Catalogo de los manuscritos existentes en la Biblioteca universitaria de Valencia, Valence, 1913, I, est exacte: 507.

(14) Cf. par exemple, T. De Marinis, La Biblioteca (supra n. 9), Tavole 111 et 126, vol. III; 292, vol. IV.

(15) Par ex., T. De Marinis, La Biblioteca (supra n. 9), II, p. 43: notice sur le manuscrit, au sein du catalogue de la bibliothèque des rois aragonais de Naples; et P. Cherchi et T. De Roвertis, "Un inventario» (supra n. 9), p. 334 (équivalences des cotes dans les différents inventaires ou catalogues), avec toujours la cote Val. 221 au lieu de 211.

(16) L'écu aux armes du royaume de Naples soutenu par des anges ou des putti apparait sur le premier folio de la plupart des manuscrits reproduits sur les nombreuses planches des volumes de T. De Marinis, La Biblioteca (supra n. 9).

(17) J'exprime toute ma gratitude à Patricia Stirnemann (IRHT) d'avoir pris le temps de regarder ce manuscrit avec moi pour essayer de le dater, ainsi qu'à F. Avril qu'elle a consulté pour moi. 
1481 quand la bibliothèque royale fut remise en gage à un banquier napolitain, lors d'un emprunt du roi pour repousser les Turcs débarqués à Otrante $\left({ }^{18}\right)$.

Après avoir séjourné à Naples et avoir été intégré à la bibliothèque royale par sa reliure et le décor du premier folio, il fut apporté à Valence dans les circonstances que nous avons vues. Il passa ensuite au monastère de S. Miguel de los Reyes, fondé par Ferdinand en 1536, puisqu'il porte sur son premier folio une attestation de son appartenance à la bibliothèque de ce monastère: «Es de la libreria de San Miguel de los Reyes» (voir pl. 40). Il y est probablement entré en 1550, au moment du legs du duc de Calabre; car dans l'inventaire de ce legs, on retrouve la trace du manuscrit de Cassiodore couvert de cuir vert: "Cassiodorus de anima, de mano, en pergamino, cubierto de cuero verde». En 1825, à la suite de la suppression des congrégations religieuses, ce qui restait de la bibliothèque du duc de Calabre fut inclus dans la bibliothèque universitaire $\left({ }^{19}\right)$.

Accursius a donc pu voir et lire le manuscrit $\beta$ à Valence. Mais s'est-il rendu dans cette ville ? Oui, on le sait par ses écrits: il a séjourné là à deux reprises, quand il suivait la cour de Charles Quint, d'abord en 1525, après ètre passé par Barcelone et avant de se diriger vers Tolède; puis de nouveau en avril-mai 1528, au terme d'un long périple dans de nombreuses villes de toute l'Espagne, où il a cherché, lu et transcrit des inscriptions. Son appartenance à la Cour de Charles Quint lui a certainement facilité l'accès aux collections du duc de Calabre, qui devait son titre de vice-roi et ses rentes à l'empereur $\left({ }^{20}\right)$.

\section{Le travail d'édition d'Accursius}

Du point de vue historique et géographique, rien n’interdit donc que ce manuscrit, conservé à la bibliothèque universitaire de Valence, soit celui dont s'est principalement servi Accursius pour établir le texte de son édition. Toutefois, comme le pensait Mommsen, un tel savant n'a pu se contenter d'établir son texte d'après un seul manuscrit, aussi excellent soit-il, malgré ce que laisse entendre le titre donné à l'ouvrage: libri XII, item de anima liber unus recens inventi et in lucem dati:

«À un homme formé par ses nombreux voyages, qui mettait sa science et son activité à chercher des textes aussi bien sur les pierres que dans les bibliothèques, n'ont pu échapper facilement les exemplaires de Cassiodore conservés çà et là dans les bibliothèques en ce temps-là; et, quand bien mème il a pu dédaigner ceux qui étaient communs et mutilés au profit d'un seul exemplaire excellent et complet qu'il aurait vu, cette édition ne peut que difficilement avoir été réalisée d'après un livre unique. Il n'y en a pas de tel, du moins parmi ceux que nous avons lus $\left(^{21}\right)$.

(18) H. Omont, «Inventaire de la bibliothèque de Ferdinand Ier d'Aragon roi de Naples (1481)», Bibliothèque de l'Ecole des Chartes, T. LXX, 1909, pp. 1-15.

(19) P. Cherchi et T. De Robertis, «Un inventario» (supra n. 9), p. 110.

(20) Sur Accursius, Dizionario biografico degli Italiani, vol. 1, «aaron-albertucci», Rome, 1960, article "Accursio» de A. Campana, p. 126-132. Pour le détail de son périple espagnol, cf. Corpus Inscriptionum Latinarum, II, 1869, p. VII-VIII (E. Hübner).

(21) T. Mommsen, Variae (supra n. 1), Préface, p. CX: uirum multis peregrinationibus exercitatum et bibliothecarum non minus quam lapidum scriptorum indagatorem doctum et strenuum, neque [primi secundique ordinis] exempla Cassiodorana [neque sexti] ea aetate in bibliothecis passim adser- 
De fait, non seulement le texte de l'editio princeps n'est pas identique à celui du manuscrit de Valence - j'ai relevé quatre pages de divergences pour le livre I -, ce qui montre qu'Accursius s'est servi d'autres manuscrits, les a comparés et a choisi; mais il y a d'autres différences plus considérables dans la présentation. Ainsi, on lit dans l'édition une adresse avant chaque lettre, comme dans les manuscrits les plus anciens, alors que ces adresses ont été complétées ou le plus souvent remplacées par des rubriques explicatives dans $\beta$, avec suppression du nom du destinataire, le plus souvent. On peut ainsi lire, pour I, 2 :

(Accursius) Theoni u. s. Theodericus rex

( $\beta)$ Redarguitur quidam de negligentia et tarditate commissis circa ornatum uestimentorum regalium Rubrica (autre ex. pl. 41)

Par ailleurs, le texte d'Accursius ne comporte pas d'interpolations, alors que $\beta$ en présente un certain nombre: c'est ainsi que, dans certaines lettres, on passe sans prévenir au texte d'une autre lettre et que le bon texte a ensuite été inscrit par une autre main dans la marge $\left({ }^{22}\right)$. Notre éditeur disposait donc d'un ou de manuscrits plus anciens et de meilleure qualité que $\beta$, qui lui ont permis d'éviter les interpolations et les inversions et d'aligner les livres dans le bon ordre, ce qui n'est pas le cas dans $\beta$, qui ne compte que 11 livres. Les douze y sont en fait présents, selon la répartition suivante $\left({ }^{23}\right)$ :

préface: fol. $1 \mathrm{r}$ à $2 \mathrm{r}$

livre $1=\mathrm{I}$ : fol. $2 \mathrm{r}$ à $16 \mathrm{v}$. Complet.

livre $2=\mathrm{II}$ : fol. $16 \mathrm{v}$ à $30 \mathrm{r}$. Complet.

livre $3=\mathrm{III}$ : fol. $30 \mathrm{r}$ à $45 \mathrm{r}$. Complet.

livre $4=\mathrm{IV}, 1$ à 39 : fol. $45 \mathrm{r}$ à $53 \mathrm{v}$. Incomplet.

livre $5=$ VIII : fol. $53 \mathrm{v}$ à $68 \mathrm{v}$. Complet.

livre $6=\mathrm{IX}$ : fol. $68 \mathrm{v}$ à $81 \mathrm{r}$. Complet.

livre $7=\mathrm{X}$ : fol. $81 \mathrm{r}$ à $93 \mathrm{r}$. Complet + la praefatio chartarum du livre XI.

livre $8=\mathrm{XI}$ : fol. $93 \mathrm{r}$ à $106 \mathrm{v}$. Complet.

livre $9=$ XII, $1+$ IV 40 à $51+$ V complet: fol. $106 \mathrm{v}$ à $125 \mathrm{r}$.

livre $10=\mathrm{VI}$ : fol. $126 \mathrm{r}$ à $137 \mathrm{v}$. Complet.

uata facile latere potuerunt, et quamquam fi eri potest, ut prae uno eximio sibi uiso et pleno uulgaria mutilaque spreuerit, ipsa editio uix ad unicum librum confi ci potuit, certe inter eos quos nos euoluimus talis nullus est.

(22) Ainsi, la fi n de I, 25, 3 est remplacée par une partie du texte I, 34 et vice versa, sans que rien ne prévienne le lecteur. Le bon texte ajouté par une $2^{\mathrm{e}}$ main dans la marge a apparemment été copié sur un autre manuscrit et l'on a donc deux versions de ces textes déplacés, qui sont différentes: par exemple, en I, 34, 1, on lit de ceteris dans la marge, mais de exteris dans le passage placé en I, 25 (Pour un autre exemple voir pl. 41).

(23) Le catalogue ne présente pas le relevé des livres (M. Gutiérrez del Caño, Catalogo, supra n. 13). 
livre 11 = VII, 1 à 41 + livre XII sauf le $1^{\text {er }}$ texte: fol. 137v à 160r. Explicit du livre XII. De anima: fol. $160 \mathrm{r}$ à $173 \mathrm{v}$

Rubriques: fol. $175 \mathrm{r}$ à $184 \mathrm{r}$

Non seulement l'ordre est bouleversé, mais il manque aussi la fin du livre VII (textes 42 à 47), comme dans la plupart des exemplaires des Variae jusqu'au XIV ${ }^{\mathrm{e}}$ s., alors qu'Accursius a publié le tout. Ce manuscrit fait donc partie des volumes mutilés que mentionne Mommsen; Accursius a complété son texte gràce à d'autres. C'est net aussi dans les livres X, XI et XII, où les convergences avec $\beta$ se raréfient considérablement (cf. les annexes) : Accursius a là visiblement privilégié d'autres manuscrits. Au tout début du livre VI, il se sert comme ailleurs de $\beta$, puis il le met de côté à partir de VI, 7 et jusqu'à la fin du livre VII pour s'appuyer sur un ou d'autres manuscrits qu'il reste à identifier: on peut supposer qu'il a pris comme modèle principal un exemplaire contenant l'intégralité du livre VII.

Le fait de disposer du manuscrit qui manquait permet de mieux évaluer l'apport d'Accursius, puisque $\beta$, s'il est intéressant, n'est pas excellent, alors que l'édition est sérieuse et de bonne qualité. Accursius a donc réalisé un véritable travail d'éditeur, à l'aide d'autres manuscrits, dont au moins un qui contenait les textes manquants dans $\beta$, à savoir VII, 42 à 47. Il faudrait un travail approfondi et portant sur les autres livres du recueil pour essayer de déterminer quels manuscrits il a utilisés. Ce travail d'édition - et non de simple copie, comme sembleraient l'indiquer le titre et la préface cités plus haut - correspond du reste bien à ce que l'on sait de cet humaniste, qui a passé sa vie à collecter des inscriptions au cours de ses nombreux voyages et à chercher des manuscrits pour corriger le texte des auteurs de l'antiquité romaine. Il insiste ainsi, dans la Préface de son édition des Variae, sur le fait qu'il consacre sa vie à l'étude: in studiis aetatem ago. Il précise également, de façon assez contournée, qu'il ne s'est pas contenté de trouver ces livres des Variae, mais qu'il a aussi travaillé à les rendre meilleurs: "Nous avons pris l'habitude de mêler notre personne et notre nom non pas seulement à ce qui est déjà presque nôtre quand nous l'avons trouvé, mais bien plus encore à ce que nous avons quelque peu amélioré» $\left({ }^{24}\right)$. De ce souci témoigne aussi le titre de son édition d'Ammien Marcellin, datant, tout comme celle des Variae, de 1533: Ammianus Marcellinus mendis quinque millibus purgatus et libris quinque auctus ultimis nunc primum inuentis. Il se vante également d'avoir corrigé 363 erreurs dans le De anima de Cassiodore, 700 dans Claudien ex vetustis exemplaribus, dum Germaniam Sarmatiasque nuper peragramus ... inter equitandum (Diatribae, A 4r) et plus de 60 dans l'editio princeps de Rutilius Namatianus (Diatribae, I 6r).

Il est difficile de savoir quels autres manuscrits a utilisés Accursius et par conséquent d'évaluer précisément son intervention d'éditeur. Voici pourtant quelques exemples, tous puisés dans la préface ou le livre I: il est d'abord, tout simplement, revenu à une orthographe plus proche de l'orthographe antique (par exemple, pedisequae pour pedisseque ou pedisecce, dans la préface, §6; ou les noms de savants de I, 45, 4, Pythagoras, Ptolemaeus, Nicomachus, Aristotiles, qui sont déformés ou orthographiés différemment dans les ma-

(24) Préface d'Accursius: ... non his solum, quae iam nostra pene sunt, dum inuenerimus, sed quae parum meliora etiam fecerimus, nos nostrumque nomen inserere, consuetudo inoleuerit. 
nuscrits) $\left({ }^{25}\right)$. Il semble ètre parfois intervenu ponctuellement pour revenir à un latin plus classique que celui des manuscrits, voire que celui de Cassiodore ! Il introduit ainsi la préposition a avant prioribus dans la formule suivante: Pudor est degenerasse prioribus. En I, 2,6 , il suit l'ordre des mots propre à $\beta$ tout en restituant l'intégralité de ces mots tronqués: per la. prae. $\beta^{1} /$ per latores praesentium Acc / per praesentium latores (ou portitores) ailleurs.

On peut aller plus loin et essayer de repérer des conjectures qui lui seraient propres; c'est toutefois délicat, puisqu'il a pu utiliser un manuscrit que nous ne connaissons pas. En I, 45, 4, la leçon ausoniis, que je n'ai rencontrée nulle part, semble être une conjecture due à la leçon de $\beta$ et de quelques autres manuscrits qu'il a adoptée dans la phrase précédente et parallèle: Translationibus enim tuis Pythagoras musicus, Ptolemaeus astronomus leguntur Itali (Italiae L R $\beta$ et Acc); Nicomachus arithmeticus, geometricus Euclides audiuntur Ausonii (Ausoniis Acc). En I, 45, 6, il semble bien s'ètre lancé dans une conjecture sur laquelle il est ensuite revenu dans ses errata: en effet, là où la plupart des manuscrits donnent

Diomedes in aere grauius bucinat/buccinat

et $\beta \quad$ Diomedes in aera gracius bucinant,

Accursius écrit Diomedis in aere grues buccinant,

avant de corriger en Diomedeae in aere grauius buccinant, gardant le verbe au pluriel de $\beta$.

En I, 32, 4, au lieu de ut is qui praesumpserit, uetitam ipse iudicetur quaesisse discordiam, il écrit ut is qui praesumpserit uetita, ipse iudicetur quaesisse discordiam, ce que je n'ai lu nulle part (on lit un neutre pluriel uentura dans le mauvais manuscrit de Ljubljana, 4 (55)). En I, 2, 1, il a supprimé un nos superflu qu'on lit partout: sacrae uestis operam, quam [nos] uoluimus necessaria festinatione compleri. En I, 18, 1, il écrit aequitatem populo dicere plutòt que aequitatem populi dicere, que donnent les meilleurs textes ainsi que $\beta$, mais qui pose un problème de construction $\left({ }^{26}\right)$, résolu dans d'autres manuscrits sous la forme populis dicere ou populis dare. Il est probable qu'Accursius a suivi ce type de manuscrits pour le datif, mais a préféré le singulier de $\beta$. En I, 29, il est le seul à écrire inducantur, là où l'on lit indicantur: avant Mommsen, qui écrit infligantur par conjecture, il a ressenti le besoin de modifier la phrase, qui n'est pas facile à interpréter $\left({ }^{27}\right)$.

Si l'on regarde les loci selecti de Mommsen, pour lesquels ce dernier a passé en revue presque tous les manuscrits dont il connaissait l'existence, il apparaît que le texte d'Accursius est, en I, 9, 1 (p. CIL), le seul à écrire debetur au lieu du debent de la plupart des manuscrits, y compris $\beta$; de même, dans la préface, $\$ 5$ (p. CXXXII), il est seul à écrire possimus, où l'on lit généralement possumus: Inter haec cur requiritis dictationis eloquium, ubi copiam uix possumus habere sermonum ? Il a de mème visiblement pris le parti de corriger aestimatur (ou extimatur) que présentent tous les manuscrits en aestimat (Préface, \$10) par souci

(25) Mais pas entièrement: il écrit patritius ; preciosus ; solatium. Les -ae- sont parfois écrits -e-.

(26) Oportet uos colere et obseruare iustitiam, qui aequitatem populo dicere suscepistis... (texte d'Accursius).

(27) Quapropter deuotio uestra praesenti iussione commonita terrarum spatia, quae ueredis antea licuerant, mutationibus suis a possessore uendicata restituat, ut nec illis paruo spatio inducantur damna et sitis recuperata suffi ciant(texte d'Accursius). 
de compréhension de la phrase. On peut supposer sans trop de risques que ce sont bien là trois autres de ses conjectures.

On ne peut savoir s'il a lu quelque part, pour le destinataire de la lettre I, 40, le nom Assius, généralement nommé Osuin $\left({ }^{28}\right)$, ou s'il l'a conjecturé d'après toutes les variantes que l'on peut en lire; il en va de même pour le destinataire de I, 36, Theoriolus, au lieu de Ferriolus $\left({ }^{29}\right)$ : on lit Theriolus en Vaticano, BAV, Chigi A. VII. 217 (V), et il est donc plutôt plausible qu'il ait trouvé cette variante dans un manuscrit. A-t-il lu quelque part nesciunt $a b$ aere, qui est visiblement une erreur (on lit aere quelques mots auparavant), au lieu de nesciunt habere en I, 45, 6, ou s'est-il trompé $\left({ }^{30}\right)$ ? Il y a de fait quelques rares erreurs, coquilles ou oublis dans le texte, comme le montre la liste d'errata qu'il a établie. On peut du moins interpréter ainsi quelques passages surprenants: par exemple, forte est omis en I, 41,1 , alors qu'il figure dans tous les manuscrits.

Il est plus facile de comprendre la manière dont il s'est servi de $\beta$ : il n'en a pas retenu les leçons aberrantes par le sens - I, 4, 7, elatus fauore fortunae in cothurnum que donne la plupart des manuscrits, et non elatus fauore fortunae in cornu $\beta$ - ou par la syntaxe (I, 27, 4, reuerentissimo senatori et non reuerentissimos senatori que donne $\beta$ seul). Il remédie aux omissions de son modèle, comme pantomimum en I, 33, 1 ou aequitas en I, 36, 2 ou encore audebit ergo seditiosos et a disciplina publica deuiantes nostra auctoritate en I, 44, 4. Par ailleurs, il suit presque toujours $\beta$ pour l'ordre des mots (sur 89 points communs entre Accursius et $\beta$ relevés dans le tableau ci-dessus, 29 portent sur l'ordre des mots, soit un tiers) et souvent pour les formes des noms propres rares ou peu connus $\left({ }^{31}\right)$. Pour le reste, c'est beaucoup moins systématique. On peut toutefois remarquer que, quand il diverge de $\beta$, ce n'est généralement pas pour préférer une leçon isolée, mais plutôt une leçon bien attestée.

\section{Singularité du manuscrit de Valence}

Ce manuscrit, loin d'être excellent, a cependant une certaine valeur: il n'appartient en effet à aucune des autres grandes familles que l'on peut repérer (celles de $M$, de $X$, de $E F$, de $K$ etc.) C'est bien pourquoi Mommsen cite l'édition d'Accursius, à travers laquelle transparaît cette originalité. $\beta$ présente toutefois des points communs avec certaines familles, celle de $K$ et celle de $E F$. Il se trouve que ces derniers manuscrits proviennent tous d'Italie, Italie du Nord semble-t-il pour $K$, du centre pour les deux autres, et probablement de Florence pour Vaticano, BAV, Chig. A. VII. 217, très proche de $E$ et $F$ et que j'appelle $V$. Il faut ajouter que le manuscrit de Wroclaw déjà cité (cf. note 2), qui a des points communs nombreux avec $\beta$ d'après les leçons relevées par Mommsen, a été copié à Avignon par un

(28) Ou Asuin, Asum et autres variations.

(29) On lit aussi Ferriosus, Serriolus, Terrilius et Terriolus orthographié de différentes façons.

(30) Diomedes in aere grauius bucinat, aeneus anguis insibilat, aues simulatae fritinniunt et quae uocem propriam nesciunt habere, dulcedinem probantur emittere cantilenae (texte de Mommsen).

(31) En I, 14, 1, il corrige le archathaliensibus aberrant de $\beta$, en faisant du $a$ une préposition, comme dans tous les autres manuscrits, mais il garde l'orthographe médiévale chargée en $h$ de $\beta$, ce qui donne a chathaliensibus (a cat(h)aliensibus, a tataliensibus ailleurs). 
Padouan en 1358, le notaire Ugerius. L'ordre dans lequel les livres y sont présentés diffère et l'ordre des textes y est plus bouleversé que dans $\beta\left({ }^{32}\right)$. Ils n'ont vraisemblablement pas été copiés l'un sur l'autre, d'autant plus que le modèle se trouvant à Avignon était luimème bouleversé, d'après les annotations d'Ugerius (cf. Mommsen, p. xc). Il n'en reste pas moins tout un faisceau de données qui convergent vers le centre et le nord de l'Italie, où l'on peut supposer que se trouvait le modèle sur lequel $\beta$ a été copié.

$\beta$ présente de surcroît parfois quelques leçons communes avec la famille de $X Y$ et $\varkappa\left({ }^{33}\right)$, et notamment des leçons propres à cette famille: par exemple, en I, 35, 2, modificat ( $\mathrm{X} \varkappa \mathrm{Y}$ $\beta$ ) au lieu de modificatus (ailleurs et chez Accursius); et en I, 37, 1 nefarium $\left(\varkappa \beta^{1}\right)$ au lieu de necessarium (ailleurs et chez Accursius). Les manuscrits de cette famille sont français et anglais et sont bien antérieurs à $\beta$; mais l'un de ceux qui se trouvent aujourd'hui à Florence (Laur., Strozzi, 23, que j'appelle $\varphi$ ), écrit probablement dans cette région (au XIII ${ }^{\mathrm{e}}$ s. pour Mommsen, dans la $1^{\text {ère }}$ moitié du XIII ${ }^{\mathrm{e}}$ s. pour P. Stirnemann, au XII ${ }^{\mathrm{e}}$ pour Halporn, comme pour le catalogue de la bibliothèque $\left({ }^{34}\right)$ ), témoigne qu'il existait une branche italienne de cette famille, par laquelle $\beta$ a pu ètre contaminé. De plus, une deuxième main a parfois remplacé des leçons de $\varphi$ propres à la famille de $X$ par des leçons qu'on trouve dans $\beta$. Par exemple, en I, 4, 4, entre les mots creuit conuersationis une seconde main a intercalé un tantum propre à $\beta$; en I, 2, 7, ut est hominibus devient ut est mos hominibus. Le plus souvent, ces leçons figurent aussi sous forme d'ajouts en $\beta$, comme dans les quelques exemples suivants pris uniquement dans les trois premiers textes du livre I:

\begin{tabular}{|l|l|l|}
\hline Variae & Texte de la $\mathbf{1}^{\text {ère }}$ main & $\boldsymbol{\varphi}^{2}=\beta^{2}$ \\
\hline $\mathrm{I}, 1,6$ & latores & latoribus \\
\hline $\mathrm{I}, 3,3$ & uagarentur & uel nutarent \\
\hline $\mathrm{I}, 3,4$ & sub deuotione & sub ipsa deuotione \\
\hline $\mathrm{I}, 3,4$ & inauarus & iam uerus \\
\hline $\mathrm{I}, 3,6$ & ordinatione & al. gubernatione \\
\hline
\end{tabular}

(32) L'ordre de $\beta$ correspond plutòt à celui de Wien, Oest. Nat., 924, qui a également le mème incipit que $\beta$. La catalogue Tabulae codicum manu scriptorum praeter Graecos et orientales in bibliotheca Palatina Vindobonensi asseruatorum, 1864, rééd. 1965, le date du XIII s., tout comme Mommsen, mais J. W. Halporn, Liber Magni Aurelii Cassiodori Senatoris De anima, 1973 (CCSL $96)$, p. 527, introduction) préfère le $\mathrm{XV}^{\mathrm{e}} \mathrm{s}$.: il serait alors postérieur à $\beta$.

(33) $X=$ Montpellier, Bibl. méd., 294, écrit dans la région champenoise, vers 1180-1200, d'après P. Stirnemann (XII ${ }^{\mathrm{e}} \mathrm{XIII}^{\mathrm{e}}$ s. pour le Catalogue général des manuscrits des bibliothèques publiques des départements, I, 1849); $Y$ = Rouen, Bibl. mun., 490, écrit à l'abbaye de Lyre à la fi n du XII ${ }^{e}$ siècle, cité par le catalogue du XII ${ }^{e}$ s. = Paris, BN, Lat. 4221, fol. 164-167 (Catalogue général des manuscrits des bibliothèques publiques de France, I, Rouen, t. II, 1888, p. 382); $\varkappa=$ Cambridge, Trinity Coll. Lib., 1341 (O.7.13), comportant l'ex libris de l'abbaye de St-Albans en Angleterre (ex libris S. Albani ex dono magistri Simonis, abbé de 1166 à 1183).

(34) A. M. Bandini, Bibliothecae Leopoldinae Laurentianae Catalogus manuscriptorum, II, 1899, p. 323. J. W. Halponn, Liber Magni Aurelii (supra n. 32). 
Les points communs sont donc assez nombreux et frappants pour qu'on puisse en déduire que ces corrections ou propositions ont été copiées les unes sur les autres, ou qu'elles proviennent du mème manuscrit, qui se trouvait en Italie centrale. Par conséquent, elles seraient antérieures au départ de $\beta$ pour l'Espagne et Accursius les aurait ensuite eues sous les yeux, sans en tenir jamais compte, semble-t-il. Par exemple, en I, 41, 1, $\beta^{1}$ a écrit ditauit, comme les meilleurs (LRPD), Accursius dictauit, alors que $\beta^{2}$ a écrit designauit, comme les autres.

\section{Valeur du manuscrit: quelques leçons rares}

L'intérèt de notre manuscrit tient aussi à ce qu'il comporte des leçons rares $\left({ }^{35}\right)$, dont certaines sont particulièrement intéressantes. C'est le cas pour deux passages du livre I: tous les deux se trouvent dans l'avant-dernier texte, I, 45, qui est l'un des plus longs et comporte des passages allusifs qui ne sont pas toujours élucidés. Par la voix de Cassiodore, Théodoric y prie Boèce de faire envoyer deux horloges au roi des Burgondes, Gondebaud, à la demande de celui-ci. L'une sera une horloge hydraulique, l'autre un cadran solaire. Ce texte est enrichi de digressions, qui portent notamment sur les merveilles réalisées par l'art de la mécanique (mechanisma): c'est grâce à celle-ci qu'a pu voler Dédale. Il est bien connu aussi, ajoute Cassiodore, «qu'elle fit pendre sans aucune attache un Cupidon (Cupidinem) de fer dans le temple de Diane, qu'elle fait aujourd'hui chanter des objets muets, vivre des matières inanimées, se mouvoir ce qui est immobile» $\left({ }^{36}\right)$. Tous les manuscrits portent cupidinem, sauf $\beta$, sur lequel on peut lire cuspidem, dans le texte copié en bas de la page pour remplacer l'interpolation qui se trouve indùment à cet endroit-là (voir pl. 41) $\left({ }^{37}\right)$. Cette leçon ne se lit ailleurs que sur l'autre manuscrit qui se trouve à Valence $\left({ }^{38}\right)$, plus tardif et qui présente un certain nombre de points communs avec $\beta$, nous y reviendrons.

S'agit-il d'une conjecture ou d'une leçon copiée sur un autre manuscrit ? L'adjectif épithète de cuspidem, qui devrait ètre accordé au féminin, est curieusement au masculin, ferreum ; mais le manuscrit $D$, d'une toute autre famille $\left({ }^{39}\right)$, présente la leçon ferream, qui pourrait ètre une trace d'un ferream cuspidem lu ferream cupidinem par $D$ ou son modèle, corrigé en ferreum cupidinem dans les autres manuscrits. Quoi qu'il en soit, il me semble bon de retenir cuspidem plutôt que Cupidinem: le rapport entre un épieu et Diane est plus

(35) Exemples: préface, 14, impolitas, comme seulement $L$ et $R$ (et probablement $K$ (folio manquant), puisqu'on le lit dans le mauvais manuscrit de Ljubljana, 4 (55), $\mathrm{XV}^{\mathrm{e}}$ s., qui descend de $K$ et que Mommsen ne connaissait pas).

(36) Var. I, 45, 10: haec ferreum Cupidinem in Dianae templo sine aliqua illigatione pendere; haec hodie facit muta cantare, insensata uiuere, immobilia moueri.

(37) Le passage interpolé provient de I, 38; le texte de I, 45 remplacé par l'interpolation se trouve lui en I, 30 et on y lit cupidinem.

(38) Dans les archives de la cathédrale, 252 (183): deux manuscrits écrits sur papier filigrané sont reliés dans le même volume: le nôtre est le second, que je nomme $\chi$. Les annotations de la seconde main pourraient avoir été copiées sur Vaticano, BAV, Chig. A. VII. 217 cité plus haut, sous le nom de $V$. $\chi$ est proche de $\beta$, mais il n'en est pas une copie. Cf. infra, «Postérité du manuscrit» p. 152-153.

(39) Il proviendrait d'Allemagne, d'une région limitrophe avec la France, et daterait du premier quart du XIII s., d'après P. Stirnemann. 
étroit qu'entre cette déesse et Cupidon. Par ailleurs, on lit que ce supposé Cupidon pend en l'air sans câble. Par quel moyen ? S. J. B. Barnish suggère qu'il était peut-être suspendu entre deux masses opposées de pierres magnétisées cachées dans la voùte $\left({ }^{40}\right)$. Il est avéré qu'on utilisait la puissance des aimants dans des temples: Claudien, dans l'un de ses petits poèmes, intitulé Magnes, évoque un temple de Mars et de Vénus dans lequel se trouvait leur statue, celle de Mars en fer, celle de Vénus en pierre magnétique: pour représenter leur union au cours d'une cérémonie, Mars, attiré par l'aimant, s'avançait vers Vénus immobile et l'enlaçait $\left({ }^{41}\right)$. Dans notre texte, il s'agit de suspendre en l'air et non simplement d'attirer et, quel que soit le procédé utilisé - on peut penser à des fils peu visibles -, cela semble plus facile pour un objet léger comme un épieu, que pour une statue.

La deuxième variante propre à $\beta$ se trouve dans le mème texte, un peu plus haut (\$5). Elle vient appuyer une conjecture qui m'est personnelle. On lit, à propos de l'art de la mécanique également: "Il fait tomber en cascade des eaux surgies d'en bas, [...] résonner des instruments de voix qui leur sont étrangères, il emplit leurs tubes de souffles extérieurs, si bien que ces petits (minuta) objets peuvent chanter avec art» $\left({ }^{42}\right)$. Il semble clair que, pour les instruments, Cassiodore parle des orgues, qui sont le fruit de la mécanique. Pourquoi les qualifier de minuta? Les manuscrits nous offrent plusieurs lectures de ce mot dont les nombreux jambages peuvent facilement induire en erreur le lecteur ou le copiste: on y lit munita (Ljubljana 4, (55) $\left({ }^{43}\right)$ ), iniuncta ( $\beta$, Accursius) et musica (Paris, BN, Lat. 2790), ce dernier sans doute par conjecture. Celle-ci a été reprise par Cujas dans ses annotations à l'édition d'Accursius $\left({ }^{44}\right)$, ce qui prouve bien que l'épithète minuta dérange. Le rapprochement avec le passage du même texte que nous avons cité plus haut à propos du temple de Diane (\$10) nous fournit, je crois, la solution: haec hodie facit muta cantare : pourquoi ne pas lire alors au $\$ 5$ muta possint arte cantare, en reprenant la même association entre muta et cantare ? Le sens est ainsi bien plus satisfaisant: «il emplit leurs tubes de souffles extérieurs, si bien que ces objets muets peuvent chanter avec art», d'autant plus que l'idée d'objets muets dotés d'une voix par la mécanique revient encore au $\$ 6:$ :un serpent de bronze siffle, de faux oiseaux gazouillent et ceux qui ne peuvent avoir une voix à eux émettent manifestement un doux chant» $\left({ }^{45}\right)$.

Or, pour revenir à notre manuscrit $\beta$, si l'on lit iniuncta dans le texte, une deuxième main a inscrit dans la marge: al. in muta, ce qui va dans le sens de mon hypothèse. On m'objectera qu'il y a moins de jambages dans muta que dans minuta. Certes, mais la confusion se trouve ailleurs: le muta du $\$ 10$ a été lu minuta par le manuscrit du Vatican déjà cité $(V)\left({ }^{46}\right)$ : on peut l'avoir pris à tort pour un abrégé de minuta.

(40) S. J. B. Barnish, Cassiodorus: 'Variae', Liverpool, 1992, p. 23.

(41) Carm. min. 29 (48).

(42) Var. I, 45, 5: Facit aquas ex imo surgentes praecipites cadere [...], organa extraneis uocibus insonare et peregrinis flatibus calamos complet, ut minuta possint arte cantare.

(43) Manuscrit du XV $\mathrm{XV}^{\mathrm{e}}$ s., cité supra, note 35, qui descend de $K$.

(44) Conjecture citée dans l'apparat de Mommsen. À propos des annotations de Cujas, voir le mème volume, p. cxi.

(45) I, 45, 6: Eneus anguis insibilat, aues simulatae fritinniunt et quae uocem propriam nesciunt habere, dulcedinem probantur emittere cantilenae.

(46) Vaticano, BAV, Chig. A. VII. 217. 


\section{Postérité du manuscrit dans la tradition espagnole des Variae}

Pour terminer, on peut ajouter que $\beta$ a eu une postérité dans la tradition manuscrite espagnole des Variae. En effet, l'un des manuscrits de l'Escurial (Bibl. mon. D.III.21, que j'appelle $\delta$ ), visiblement contaminé, a été copié entre autres sur $\beta$, comme le montrent les parallèles suivants recueillis dans le livre I, sans mème tenir compte de toutes les fois où $\beta$ et $\delta$ partagent leur lecture avec d'autres manuscrits:

\begin{tabular}{|c|c|c|}
\hline Variae & $\beta \delta$ & autres manuscrits \\
\hline $\mathrm{I}, 2,4$ & albentes comas & albentis comas \\
\hline $\mathrm{I}, 3,5$ & contentionis & contentiones \\
\hline $\mathrm{I}, 3,5$ & exasperet & exasperent \\
\hline $\mathrm{I}, 4,3$ & praecipue & praecipua \\
\hline $\mathrm{I}, 4,14$ & siciliam bruciosque & brucios siciliamque \\
\hline $\mathrm{I}, 4,18$ & nobis est & uobis est \\
\hline $\mathrm{I}, 8,2$ & facias & faciat \\
\hline $\mathrm{I}, 8,3$ & uenire deproperet & deproperet uenire \\
\hline $\mathrm{I}, 10,5$ & utiles & uiles \\
\hline $\mathrm{I}, 10,5$ & docta antiquitas & antiquitas docta \\
\hline $\mathrm{I}, 10,7$ & omnino & omnimodo \\
\hline $\mathrm{I}, 15,1$ & ut te & $u t$ \\
\hline $\mathrm{I}, 18,3$ & in memoriam & in medium \\
\hline $\mathrm{I}, 19,2$ & uobis praesenti & praesenti uobis \\
\hline $\mathrm{I}, 25,3$ & suas laudes & laudes suas \\
\hline $\mathrm{I}, 26,3$ & ecclesiam uestram & ecclesiam nostram \\
\hline $\mathrm{I}, 28,2$ & ciuitatis suae utilitati & utilitati suae ciuitatis \\
\hline $\mathrm{I}, 30,1$ & postulauit & pulsauit \\
\hline $\mathrm{I}, 30,2$ & fide mala & mala fide \\
\hline $\mathrm{I}, 30,3$ & nostra praecepta & praecepta nostra \\
\hline $\mathrm{I}, 39,2^{47}$ & aliena & alieni \\
\hline $\mathrm{I}, 39,2$ & forsitan & forte \\
\hline I, 40 & non debet per moram & per moram non debet \\
\hline $\mathrm{I}, 45,2$ & est cottidianum & cottidianum \\
\hline $\mathrm{I}, 45,10$ & hoc hodie & haec hodie \\
\hline
\end{tabular}

Ce manuscrit de Madrid semble aussi avoir intégré dans son texte des variantes apposées dans la marge de $\beta$ par une deuxième main. En voici quelques exemples, toujours puisés dans le livre I:

(47) Dans le texte emprunté à IV, 6, 3 interpolé dans plusieurs manuscrits. 


\begin{tabular}{|l|l|l|}
\hline Variae & $\boldsymbol{\beta}^{2} \boldsymbol{\delta}\left(\boldsymbol{\varphi}^{2}\right)$ & $\beta^{1}$ \\
\hline $\mathrm{I}, 3,3$ & nutarent $+\varphi^{2}$ & uagarentur \\
\hline $\mathrm{I}, 3,4$ & sub deuotione $+\varphi^{2}$ & sub ipsa deuotione \\
\hline $\mathrm{I}, 3,6$ & gubernatione $+\varphi^{2}$ & ordinatione \\
\hline $\mathrm{I}, 3,6$ & nullus enim grauanter & nullus grauanter \\
\hline $\mathrm{I}, 4,6$ & prouectorum & prosperorum \\
\hline $\mathrm{I}, 9,3$ & talibus modum imponere & talibus imponere \\
\hline $\mathrm{I}, 13,2$ & eugenium & eugenitem \\
\hline $\mathrm{I}, 16,2$ & decreuimus & iudicamus \\
\hline $\mathrm{I}, 16,3$ & supplica(n) cium & supplicum (ou supplicium) \\
\hline $\mathrm{I}, 18,1$ & dare & dicere \\
\hline $\mathrm{I}, 18,4$ & exosa societas sola & exosa societas \\
\hline $\mathrm{I}, 20,2$ & ubique possimus & ubique possit \\
\hline $\mathrm{I}, 37,1$ & ne nefarium $\left(\delta^{2}\right)$ & necessarium \\
\hline $\mathrm{I}, 37,1$ & (non) trahi & trahere \\
\hline $\mathrm{I}, 44,1$ & uestram & uestri \\
\hline $\mathrm{I}, 44,4$ & illicitis in priorem statum cuius reuocentur & illicitis \\
\hline
\end{tabular}

Certaines d'entre ces leçons se trouvent aussi, écrites par une seconde main, dans le manuscrit de Florence dont nous avons parlé $\left({ }^{48}\right), \varphi$, mais la plupart ne se lisent que dans $\beta$; c'est donc plutôt de ce dernier que $\delta$ descend. Il y a toutefois quelques leçons propres à $\varphi$ et $\delta$ (par exemple I, 2, 6: mittemus, au lieu de mittimus) qui peuvent faire supposer que le copiste s'est aussi servi d'un manuscrit de la famille de $\varphi$, entre autres (points communs également avec la famille $E F V$ ). Il semble avoir copié le décor d'un manuscrit de Padoue ou de Bologne, d'après P. Stirnemann, sans être pour autant nécessairement italien. Toutefois, s'il est, comme elle le suppose, du XIV siècle $\left({ }^{49}\right)$, il a certainement été écrit en Italie, avant le transfert de $\beta$ à Valence, ce qui expliquerait l'influence des manuscrits italiens cités. Il faudrait chercher par quel biais il est arrivé à l'Escurial pour mieux cerner son origine.

$\beta$ a également eu une influence sur les deux manuscrits sur papier qui se trouvent reliés ensemble dans les archives de la cathédrale, 252 (183): le second, que je nomme $\chi$, est proche de $\beta$ (mêmes rubriques, leçons communes), mais il n'en est pas une copie. Quant au premier manuscrit relié dans le volume, il a des points communs avec $\beta$ (mêmes rubriques, leçons communes) et $\chi$, mais il est spécifique: un souci très maladroit de corriger les Variae, de les adapter, de simplifier le vocabulaire a entraîné des erreurs et des leçons aberrantes. Provient-il d'Italie ? On peut en effet lire sur le folio 1: Ex bibliotheca Iosephi

(48) Cf. supra, au niveau de la note 34.

(49) Le catalogue le date également du XIV s.: P. G. Antolìn, Catálogo de los Códices Latinos de la Real Biblioteca del Escorial, I, Madrid, 1910. Ce catalogue ne donne aucune indication de provenance. 
Stephani episcopi Vestani decani ecclesie valentine 1590. Ce personnage originaire de Valence (Don José Esteve Juan, 1551-1603) fut, après des études à Sienne et un séjour à Rome, nommé évêque de Vesta (Foggia) par Sixte V, dans l'antique royaume de Naples, en 1586. Il y résida peu, puis devint decanus de la cathédrale de Valence, puis évêque d'Orihuela (Alicante) en 1594. Auteur de nombreuses œuvres, essentiellement de caractère juridique, il possédait une très importante bibliothèque : 1595 volumes furent expédiés au Vatican en 1605, que le pape Paul V offrit en 1608 à son neveu le cardinal Scipione Borghese, biblio-thécaire du Vatican depuis 1607 et dont les collections restèrent à la Vaticane $\left({ }^{\$}\right)$. Notre manuscrit a probablement échappé à cet envoi en Italie : il est peut-être resté à Valence, alors que le reste de la bibliothèque se trouvait à Orihuela à la mort de l'évêque. S'il a été écrit en Italie, où l'évêque aurait pu le trouver, c'est avant l'arrivée de $\beta$ à Valence. Seule sa datation permettrait de répondre $\left({ }^{51}\right)$. Halporn $\left({ }^{52}\right)$ le date du $\mathrm{XV}^{\mathrm{e}}$ s., sans l'avoir vu toutefois : aurait-il alors été écrit au début du $\mathrm{XV}^{\mathrm{e}}$ siècle dans le royaume de Naples, où l'évêque de Vesta l'aurait trouvé par la suite ? Pour conclure, si $\beta$ a eu une postérité qui se trouve aujourd'hui pour partie au moins en Espagne, il semble qu'il n'y ait pas eu de tradition manuscrite espagnole des Variae, mais l'arrivée, par diverses voies, de manuscrits provenant essentiellement d'Italie.

Le manuscrit 211 conservé à la bibliothèque universitaire de Valence est intéressant à plus d'un titre: par son histoire mouvementée d'abord, liée à l'histoire du royaume aragonais de Naples; par les traces qu'a laissées cette histoire sur le volume, notamment le changement de décor de son premier folio au moment de son entrée dans la bibliothèque royale. Comme nous l'avons montré, son intérêt tient également au rôle de premier plan qu'il a joué dans l'editio princeps d'Accursius: sa découverte est présentée comme une «invention» par ce dernier et il faut croire qu'il avait alors quelque chose de plus que les manuscrits italiens accessibles au milieu des vicissitudes que traversait l'Italie du début du XVI ${ }^{\mathrm{e}}$ siècle, qui ruinèrent par ailleurs la première tentative d'édition, celle de Jean Cochlaeus, quelques années auparavant. Dans l'état actuel de la question, ce manuscrit influencé par plusieurs familles est inclassable et présente, sans être excellent, des particularités de texte notables et des leçons parfois intéressantes. Si l'on ne sait de quel archétype il descend, on peut en revanche repérer plusieurs volumes qu'il a influencés ou contaminés, volumes qui se trouvent également en Espagne, mais qui semblent, sous toutes réserves, provenir aussi d'Italie. La confrontation de ce texte avec celui d'Accursius permet enfin de mesurer l'importance et la qualité du travail d'édition auquel s'est livré ce savant voyageur huma-

(50) Pour une bibliographie complète, voir J. Martinez Valls, «Semblanza biográfica del Obispo de Orihuela Don José Esteve Juan (1551-1603), y sus relaciones 'ad limina'", in Anthologia annua, no 26-27 (1979-1980), Rome, Instituto Español de Historia Eclesiástica, p. 555-612.

(51) J'ai vu les deux manuscrits à Valence, mais je n'ai pu encore en obtenir ni copie, ni photo et je n'ai pas les compétences nécessaires pour les dater de façon fiable. Il me semble que le second pourrait dater du $\mathrm{XIV}^{\mathrm{e}} \mathrm{s}$. et que le premier serait plus récent. Je n'ai pas su non plus en identifier les filigranes: celui du premier m'a semblé se terminer par les lettres BVLL. Pour le second, j'ai lu TIMIVS ou TINAIVS.

(52) J. W. Halponn, Liber Magni Aurelii (supra n. 32), p. 527. 
niste $\left({ }^{53}\right)$, bien que nous ne sachions pas de quels autres manuscrits il s'est servi: travail de retour à l'orthographe antique, de remise en ordre des livres et des textes, de correction des adresses, d'élimination des lacunes, de tri des variantes et de choix parmi elles, travail de conjecture enfin.

Paris Ouest-Nanterre

Valérie Fauvinet-Ranson

\section{ANNEXE}

Sondages effectués dans les livres II à XII, confrontant $\beta$ et le texte d'Accursius, et s'appuyant sur l'apparat-critique de Mommsen: (seules les leçons propres à $\beta$ et Accursius, à l'exclusion des autres manuscrits de l'apparat, ont été retenues).

Livre II, 6 à $11: 10$ points communs en 6 textes:

\begin{tabular}{|l|l|l|}
\hline Variae & Acc $\beta$ (+ Wroclaw) & Autres mss \\
\hline II, 6,2 & tuum iure $+W$ & tuum \\
\hline II, 6,2 & potuisses probare & probare potuisses \\
\hline II, 7 & magnificentia & sublimitas (ou sapientia) \\
\hline II, 8 & necessario nos & nos necessarie \\
\hline II, 9,1 & cedere honorabiliter & honorabiliter cedere \\
\hline II, 9,2 & dari menstruum & menstruum ( ou menstruum dari K) \\
\hline II, 10,2 & mererentur & merentur (ou meretur) \\
\hline II, 10,2 & decreuimus & decernimus \\
\hline II, 10,2 & illusionem & irrisionem \\
\hline II, 10,2 & lucrum sibi & lucra sibi (ou sibi lucra KE) \\
\hline II, 11,2 & petitio $+W$ & petitione \\
\hline
\end{tabular}

Livre III, 1 à 4: 10 points communs en 4 textes:

\begin{tabular}{|l|l|l|}
\hline Variae & Acc $\boldsymbol{\beta}(+$ Wroclaw) & Autres mss \\
\hline III, 1,1 & ferocia & ferocium \\
\hline III, 1,3 & enim om. & enim (ou etenim) \\
\hline III, 2, 2 & illud in uobis & illis in uobis ou illi in nobis \\
\hline
\end{tabular}

(53) L'apport d'Accursius aux textes est mal connu: cf. par ex. A. Campana, dans Dizionario biografico (supra n. 20): "un vero giudizio sul suo contributo critico, sulla sua capacità e sui suoi procedimenti di editore, presuppone uno studio analitico e strettamente tecnico che ancora manca; solo materiali forniscono le introduzioni e gli apparati degli editori moderni» (Mommsen, et Clark pour Ammien). 


\begin{tabular}{|l|l|l|}
\hline III, 2,3 & credimus $+W$ & credidimus \\
\hline III, 3,3 & melius est $u t+W$ & melius \\
\hline III, 3,3 & henrici $+W$ & eurici $($ enrici $E)$ \\
\hline III, 3,3 & tantum $+W$ & tamen \\
\hline III, 3,3 & praesumet $+W$ & praesumit \\
\hline III, 4,2 & alarico rege & rege alarico \\
\hline III, 4,3 & protinus arma mouere & arma protinus commouere \\
\hline
\end{tabular}

Livre IV, 40 à $\mathbf{5 1}: 10$ points communs en 12 textes:

\begin{tabular}{|l|l|l|}
\hline Variae & Acc $\boldsymbol{\beta}$ (+ Wroclaw) & Autres mss \\
\hline IV $, 40,3$ & decet & debet \\
\hline IV $, 44,2$ & in tam & in causa \\
\hline IV $, 44,2$ & iustitia $+W$ & iustitiam \\
\hline IV $, 47,1$ & subiecti ueredorum & subuectio ueredorum ou subuectiuae redorum \\
\hline IV $, 47,3$ & est unius aestimanda $+W$ & aestimanda est unius \\
\hline IV, 48 & decet & debet \\
\hline IV, 48 & securitate & seueritate \\
\hline IV, 48 & ratio & natio \\
\hline IV $, 51,12$ & ut $+W$ & ubi \\
\hline IV $, 51,12$ & utiliter $+W+P^{2}$ & inutiliter \\
\hline
\end{tabular}

Livre V, 33 à 42 : dix points communs en 10 textes:

\begin{tabular}{|l|l|l|}
\hline Variae & Acc $\beta$ (+ Wroclaw) & Autres mss \\
\hline $\mathrm{V}, 33,1$ & matrimonium $+W$ & matrimonii \\
\hline $\mathrm{V}, 37,2$ & iure $+W$ & iura \\
\hline $\mathrm{V}, 37,2$ & personas certe & certe personas \\
\hline $\mathrm{V}, 38,2$ & rauennate $+W$ & rauennati (ou rauennata) \\
\hline $\mathrm{V}, 39,1$ & tranquilla dispositio $+W$ & dispositio tranquilla \\
\hline $\mathrm{V}, 39,3$ & ordinationis nostrae $+W$ & ordinationis uestrae \\
\hline $\mathrm{V}, 39,6$ & iura $+W$ & rura \\
\hline $\mathrm{V}, 40,2$ & addita & adiuncta \\
\hline $\mathrm{V}, 42,3$ & herebi & erebi $($ herbi $F)$ \\
\hline $\mathrm{V}, 42,4$ & illusionem & irrisionem \\
\hline
\end{tabular}


L'usage qu'a fait Accursius du manuscrit $\beta$ semble donc constant pour ces livres en moyenne (il faut tenir compte de la longueur variable des textes, qui explique le plus ou moins grand nombre d'occurrences).

Livres VI, 1 à 6: 8 points communs en 6 textes, puis plus de points communs à partir de VI, 7 :

\begin{tabular}{|l|l|l|}
\hline Variae & Acc $\beta$ (+ Wroclaw) & Autres mss \\
\hline VI, 1,2 & quidem $+W$ & pridem \\
\hline VI, 1,6 & per maximos & post maximos \\
\hline VI, 3,3 & potest negotia sine appellatione & sine appellatione potest negotia \\
\hline VI, 3,5 & non audeant iudices & iudices non audeant \\
\hline VI, 4,7 & est enim uis $+W$ & enim uis est \\
\hline VI, 5,3 & alienos $+W$ & et alienos (ou etiam alienos) \\
\hline VI, 6,1 & terminis prospere & prospero \\
\hline VI, 6,5 & adeptae $+W$ & $\begin{array}{l}\text { indeptae }(\text { ou indemptae ou inde cepite ou inde } \\
\text { cepit ou inceptae) }\end{array}$ \\
\hline
\end{tabular}

Livre VII, 1 à 10: seulement 1 point commun en 10 textes:

\begin{tabular}{|l|l|l|}
\hline Variae & Acc $\beta$ (+ Wroclaw) & Autres mss \\
\hline VII, 7,1 & contigit & contingit \\
\hline
\end{tabular}

Livre VIII, 1 à 3: 10 points communs en 3 textes:

Les manuscrits de Wroclaw et d'Accursius concordent toujours, d'après l'apparat de Mommsen, à partir de ce livre.

\begin{tabular}{|l|l|l|}
\hline Variae & Acc $\beta$ (+ Wroclaw) & Autres mss \\
\hline VIII, 1, 1 & patres & parentes \\
\hline VIII, 1, 3 & nobis paene uidebatur & uobis paene uidebatur (place variable de uobis) \\
\hline VIII, 1, 4 & est mihi & mihi est (ou mihi) \\
\hline VIII, 1, 4 & principis longaeui habere & principis habere longaeui (ou h. p. l.) \\
\hline VIII, 2, 2 & erigunt & exigunt \\
\hline VIII, 2, 2 & fidelitatis & felicitatis (ou ciuilitatis) \\
\hline VIII, 2, 3 & qui ex uobis & ex uobis qui \\
\hline VIII, 2, 4 & nobis progenies & uobis progenies \\
\hline VIII, 2, 5 & haec & hoc \\
\hline VIII, 3, 3 & auspice & quod auspice \\
\hline
\end{tabular}


Livre IX, 1 à 9: 10 points communs en 9 textes:

\begin{tabular}{|l|l|l|}
\hline Variae & Acc $\beta$ (+ Wroclaw) & Autres mss \\
\hline IX, 1,2 & hasdiuorum A, has diuorum $\beta$ & $\begin{array}{l}\text { hasdingorum } B \text { K (variantes dont has digno- } \\
\text { rum) }\end{array}$ \\
\hline IX, 3,2 & ruris & iuris \\
\hline IX, 4,1 & potuerint & poterint \\
\hline IX, 4,2 & qui uetatur & quod uetatur \\
\hline IX, 4,3 & fuerant & fuerunt \\
\hline IX, 6,3 & tegitur & regitur \\
\hline IX, 6,6 & tui & tua (ou talia) \\
\hline IX, 7,5 & permittitur & praemittitur \\
\hline IX, 9,1 & bellicum & bellicum uitasse \\
\hline IX, 9,1 & impietate & iniquitate \\
\hline
\end{tabular}

Livre $\mathbf{X}, 1$ à 10: seulement 2 points communs en 10 textes:

\begin{tabular}{|l|l|l|}
\hline Variae & Acc $\beta(+$ Wroclaw $)$ & Autres mss \\
\hline $\mathrm{X}, 6,6$ & qui etiam & etiam qui (et qui et variantes) \\
\hline $\mathrm{X}, 7,5$ & reliquit & relinquit \\
\hline
\end{tabular}

Livre XI, 13 à 34: seulement 4 points communs en 22 textes:

\begin{tabular}{|l|l|l|}
\hline Variae & Acc $\beta$ (+ Wroclaw) & Autres mss \\
\hline $\mathrm{XI}, 13,4$ & huius & huiusmodi \\
\hline $\mathrm{XI}, 14,3$ & huic & hinc \\
\hline $\mathrm{XI}, 16,1$ & conscendere & descendere ou condescendere \\
\hline $\mathrm{XI}, 34$ & nos & uos \\
\hline
\end{tabular}

Livre XII, 1 à 10: seulement 3 points communs en 10 textes:

\begin{tabular}{|l|l|l|}
\hline Variae & Acc $\boldsymbol{\beta}(+$ Wroclaw) & Autres mss \\
\hline XII, 2, 3 & discessit & discesserit \\
\hline XII, 4, 6 & robore & rubore \\
\hline XII, 9,1 & potentium & petentium \\
\hline
\end{tabular}

